\title{
Fundamentos epistemológicos para el desarrollo de investigaciones cualitativas en educación
}

\section{Epistemological foundations for the development of qualitative research in education}

\author{
Henry Juan Javier Ninahuaman*I \\ *Departamento Académico de Educación, Facultad de Educación, Universidad Nacional Autónoma Altoandina de Tarma (UNAAT), Tarma - Perú.
}

\begin{abstract}
Resumen
A través de la teoría fundamentada se llega a la teoría sobre los fundamentos epistemológicos para el desarrollo de las investigaciones cualitativas en educación. Desde la recolección de datos, codificación, delimitación de la teoría, el lugar en la literatura, la comunicación de los resultados y la teoría formal sustantiva. Llegando a la conclusión: los fundamentos de las investigaciones cualitativas son las formas de conocer y hacer conocer la realidad educativa. Mediante el poder investigativo y la conceptualización de las manifestaciones de las personas en los fenómenos educativos de su momento. Para contribuir en el conocimiento profundo del problema investigativo cualitativo y solucionarlo o mejorarlo.
\end{abstract}

Palabras Claves: Ciencias de la educación, epistemología, administración de la ciencia y de la investigación, teoría fundamentada.

\begin{abstract}
Through grounded theory we arrive at the Theory of the epistemological foundations for the development of qualitative research in education. From data collection, codification, delimitation of theory, place in the literature, communication of results, and formal substantive theory. Reaching the conclusion: The foundations of qualitative research are the forms of knowing and making known the reality of education. Through the power of research and the conceptualization of the manifestations of people in the educational phenomena of their time. In order to contribute to a profound knowledge of the qualitative research problem and to solve or improve it.
\end{abstract}

Keywords: Educational sciences, epistemology, science and research administration, grounded theory.

1 Correspondiencia: Henry Juan Javier Ninahuaman, hjavier@cip.org.pe 


\section{Fundamentos epistemológicos para o desenvolvimento de investigações qualitativas na educação}

\section{Resumo}

Através da teoria fundamentada se chega à Teoria sobre os fundamentos epistemológicos para o desenvolvimento das investigações qualitativas na educação. Desde a recolecção de dados, codificação, delimitação da teoria, o lugar na literatura, a comunicação dos resultados e a teoria formal substantiva. Chegando à conclusão: Os fundamentos das investigações qualitativas são as formas de conhecer e fazer conhecer a realidade educativa. Mediante o poder investigativo e a conceitualização das manifestações das pessoas nos fenómenos educativos de seu momento. Para contribuir no conhecimento profundo do problema investigativo qualitativo e solucioná-lo ou melhorá-lo.

Palavras claves: Ciências da educação, Epistemologia, Administração da ciência y da investigação, Teoria fundamentada.

\section{Fundamentum epistemológicum wiñaypaq chay huchayayta taripay cualitativas chay edukasyunpi}

\section{Uchuycha}

Chay chawpinasqapi teoría fundamentada chayanchi chay Teoría fundamentos epistemológicos kururay paqhuchayaytataripaypaq cualitativas educasyunta.Tuktupallaypachaypi yuyaykunata, codificación, delimitación de la teoría, chaypi kan literatura, chay resultado willay teoría formal sustantiva. Tukuypachayaspa: Chay fundamentos Huchayaytataripaycualitativastachaymiriqsichinriqcsichispakachkaytaeduca tivata.

Chawpinasqa atipan investigatiputa chay conceptualización rimasqantarunakunapata chay fenómenos educativospunchaumanta. Ruwanapaqchipaqyachayta chay Sasay investigativo cualitativo alintaruwanapaq.

Hatun apup simi: Ciencia de la educaciomil, epistemologiam, administracioón chay ciencia maskakuna, teoría fundamentadam. 


\section{Introducción}

Los investigadores adoptan diferentes paradigmas y posturas respecto a la forma de realizar sus investigaciones. Los fundamentos de su proceder son múltiples y pocas veces se nombran y esclarecen; en el paradigma cuantitativo los fundamentos investigativos se declaran en los autores clásicos de la escuela positivista, mientras que en el paradigma cualitativo el investigador deberá de introducirse a lo incierto, difuso y emergente sostenido en el modelo dialógico principalmente. En educación no existen estudios sobre estos fundamentos.

Se hace necesario establecer una teoría que represente al investigador sobre sus fundamentos epistemológicos para el desarrollo de investigaciones cualitativas en educación y la mejor forma de hacerlo es mediante la teoría fundamentada que establece teorías a partir de los datos del investigado en un estudio sistematizado y riguroso.

\section{Recolección de la Información}

La técnica para la recolección de datos es la habilidad del uso de recursos, puede ser la observación, entrevista, cuestionario, entre otros. Para la recopilación de la información relevante al problema de estudio; este estudio eligió la técnica del cuestionario.

El cuestionario es un instrumento de la encuesta. Pueden ser entrevistas cerradas, abiertas o mixtas. Su objetivo es reunir la información adecuada para la investigación. El instrumento seleccionado para el presente estudio fue la entrevista mixta vía google form.

Participaron docentes de posgrado de la UNCP, doctorandos y directores de las instituciones de E.I.E. "Seis de Agosto", I.E. Antenor Rizo Patrón, I.E. Ángela Moreno Gálvez, Universidad Nacional del Centro del Perú, I.E. José Carlos Mariategui, Ugel Tarma y docentes de la Universidad Nacional Autónoma Altoandina de Tarma. De estos el $62.5 \%$ de sexo masculino y $37.5 \%$ femenino, entre las edades de 37 a 56 años. Prefieren la inclinación hacia la investigación mixta (55\%), seguida por la investigación cuantitativa (33\%) y solo un $11 \%$ que prefieren la investigación cualitativa. Se recolectaron la siguiente información:

Respecto a la recolección de la información a la interrogante ¿Qué es la investigación cualitativa? se configura el siguiente concepto emergente. Es un tipo y actividad de investigación que estudia las subjetividades. Toma en cuenta las manifestaciones, expresiones, interacciones de las personas para ser investigadas científicamente, además de ser participativa e interdisciplinaria. Propone la solución de emergencias de significados, fenómenos, acciones y propuestas teóricas conectadas a la realidad educativa. Formula y reformula matrices epistémicas; prácticas de enseñanza, evaluación y aprendizaje. Lo que quiere decir que toma en cuenta la manifestación de las personas en su formulación de soluciones o mejora.

Respecto al concepto de la preferencia y acción de la investigación se configura el concepto emergente. Preferencia a la investigación mixta pero no se están haciendo en su práctica investigativa. Inclinación hacia la mixta (55\%), seguida por la cuantitativa $(33 \%)$ y solo un $11 \%$ que prefieren la cualitativa. Sin embargo, las investigaciones que realizan apuntan al mismo porcentaje a $44.4 \%$ a cualitativas y cuantitativas y solo un $1.1 \%$ a la mixta. Se puede decir que hay un deseo de realizar investigaciones mixtas pero este deseo no se realiza por el momento.

Sobre el concepto emergente de los elementos determinantes para fijar su inclinación de paradigma de investigación. El elemento determinante establece la inclinación al paradigma. Son determinados por la matriz epistémica educativa, el poder de la investigación, la aplicación de las habilidades sociales en el equipo de trabajo, el interés estudiantil, la naturaleza del objeto de estudio, el conectivismo y el momento de descubrimiento y re-descubrimiento. Para tener nuevas formas de ver, interpretar, argumentar y escribir. Se puede observar la importancia del poder investigativo desde su matriz, la forma de enseñanza-aprendizaje y el momento actual. 
Respecto a lo que más sorprende de la investigación cualitativa se tiene el concepto emergente. La investigación cualitativa sorprende a los investigadores en el significado de las transformaciones sociales. La sorpresa se da en el contexto, profundización, análisis de las personas y sus circunstancias. Para una reflexión progresista del evento, inferir, conceptualizar, y discutir los resultados con el marco teórico. La sorpresa de la investigación cualitativa radica en las personas, su análisis y sus circunstancias.

Respecto a la pregunta de pérdida de la data investigativa inesperada e irrecuperable se configura la estructura conceptual emergente. Al efecto inesperado de pérdida de la data investigativa la ciencia tomaría nuevos rumbos. Se formularía otra data con diferentes procedimientos y teorías de investigación. Con pánico por la limitación de resolver o analizar los problemas relevantes. Habría perjuicio de problemas actuales pero la ciencia y los investigadores tomarían otros caminos.

Respecto a los comentarios finales se estructura un concepto emergente. El investigador considera importante a los temas no tratados. Se centra en la investigación (momento de reflexionar y crear nuevos paradigmas y formas de investigar, nivel y sentido de conocimientos de desarrollo de proyectos) y en el instrumento (Orientación de las preguntas, iniciativa, elaboración). Para hacer llegar su opinión no contemplada en el estudio. Los investigadores consideran importante los términos de investigación y el instrumento.

El concepto emergente de lo que más sorprende de la Investigación cuantitativa es la demostración, la generalización, la estadística, el marco teórico, discusión de resultados, las gráficas, interpretación de los resultados, el análisis y la evolución de resultados.

\section{Codificación}

Se codificaron los datos. Empleando la codificación abierta de códigos en vivo comparando la respuesta a las encuestas. Para hacer surgir las categorías: poder investigativo, personas, fenómeno educativo, conceptualización, y momento actual para luego pasar a la codificación condicionante para delimitarla correctamente.

\section{Delimitación de la Teoría}

\section{Ejecutar la propuesta}

En la figura 1 se muestra la estructura de la categorización.

\section{Propiedades de las categorías}

El poder investigativo es el nivel y sentido de los conocimientos en investigación. Que emplea la profundización, reflexión, creación de nuevos paradigmas y formas de investigar con la aplicación de las habilidades e instrumentos según el interés o la naturaleza del fenómeno de estudio. Para tener nuevas formas de ver, interpretar, argumentar y escribir en la solución de emergencias de significados, fenómenos, acciones y propuestas teóricas.

Las personas son los sujetos investigadores e investigados, que se muestran con su participación, expresión, interacción y personalización; para contribuir al proceso investigativo cualitativo.

El fenómeno educativo es la causa de la investigación. Se desarrolla con la reflexión progresista, el significado de las transformaciones sociales en el contexto, el análisis de las personas y sus circunstancias con la máxima profundización posible. Para ser solucionada o mejorada. 


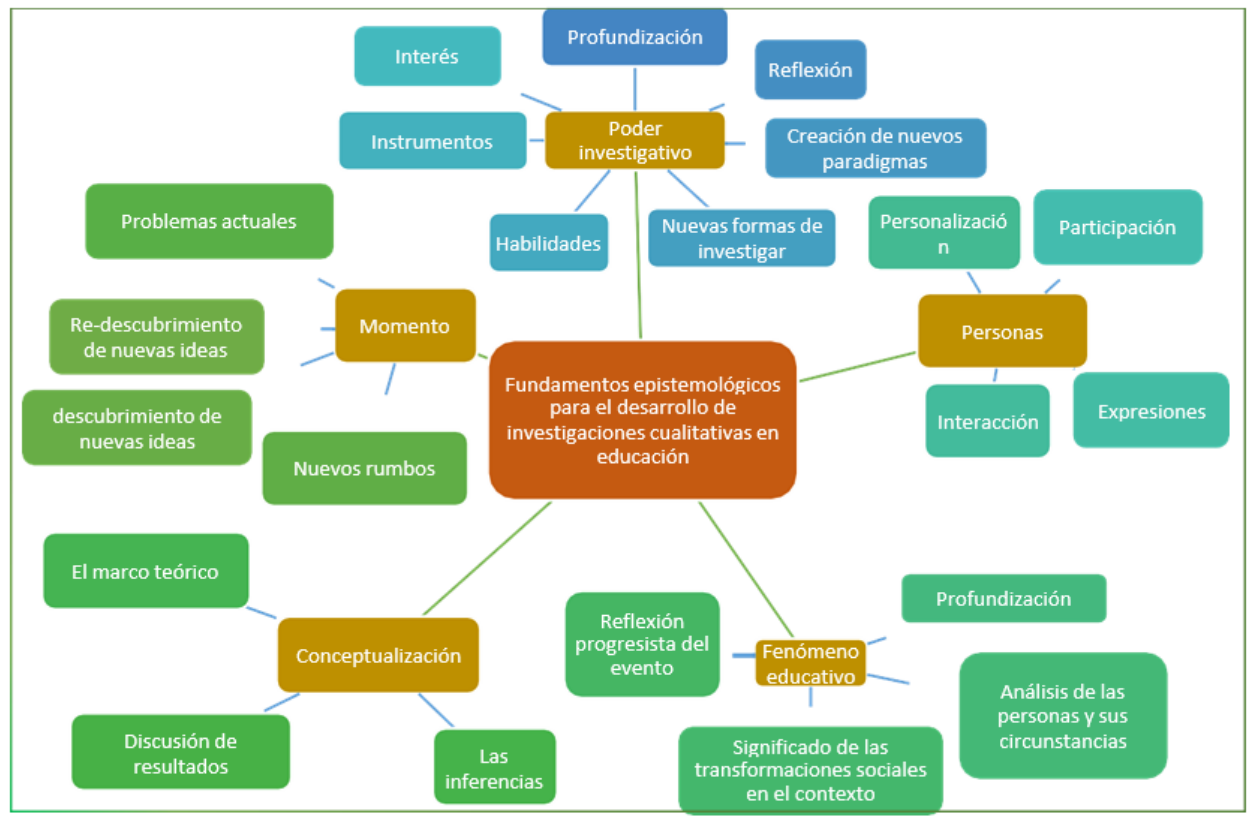

Figura 1. Estructura de la categorización de los fundamentos epistemológicos del desarrollo de las investigaciones cualitativas en educación.

La conceptualización es la representación mental de la actividad investigativa, se desarrolla desde la discusión de resultados, las inferencias y el marco teórico; para conocer profundamente el problema de investigación. Momento es el periodo de tiempo donde surge la investigación. En ese periodo se encuentran los problemas actuales y los nuevos rumbos para el descubrimiento y re-descubrimiento de nuevas ideas.

\section{Hipótesis emergente}

Las investigaciones cualitativas educativas se fundamentan en el poder investigativo y la conceptualización de las manifestaciones de las personas en los fenómenos educativos de su momento.

\section{El lugar de la literatura}

La teoría fundamentada es un instrumento conceptual de comparación constante. Que, desde la recolección de datos, codificación, delimitación de la teoría, el lugar en la literatura, la comunicación de resultados llega a la teoría formal con la que se representa el descubrimiento verificado del fenómeno educativo generador. Para comprender el significado y configuración del sentido que adquiere la realidad educativa emergente (Klimovsky et al., 1971; Vasilachis, 2006).

La investigación cualitativa tiene similitud con la cuantitativa. En la pretensión de conocer y hacer conocer la realidad, su gran poder investigativo, recolección de información para su análisis riguroso, la discusión se realiza con ideas de personas y sus hallazgos al referenciar en menor o mayor grado; quieren entender a las personas y cuentan con el interés de los investigadores, las Teorías Fundamentadas formales pueden ser generalizables (Bénard, 2016).

Sus diferencias radican principalmente en su dedicación (a sujetos o a objetos), el mínimo o nulo uso de estadística, orientación y conexión con el sujeto, la demostración subjetiva, las discusiones simultáneas e interpretación mediante la comprensión, los resultados evolucionan sin tener la necesidad de un marco teórico, se avoca a problemas holísticos y aplican las habilidades sociales por lo que se consideraría una ciencia blanda (Vanossi, 2003). 
La fundamentación epistemológica es lo que se quiere decir o explicar, determina la forma en que se conoce y estudia las culturas humanas, asignando conceptos, categorías leyes y principios; que han de ser simbolizados y transformados convenientemente (Cerrón, 2014).

\section{Teoría Formal}

Bajo el proceso de la teoría fundamentada se enuncia la teoría formal sustantiva:

Los fundamentos de las investigaciones cualitativas son las formas de conocer y hacer conocer la realidad educativa. Mediante el poder investigativo y la conceptualización de las manifestaciones de las personas en los fenómenos educativos de su momento. Para contribuir en el conocimiento profundo del problema investigativo cualitativo y solucionarlo o mejorarlo.

\section{Conclusiones}

En el ámbito doctoral los fundamentos epistemológicos para la investigación son trascendentes al punto de determinar la forma de investigar. La codificación axial y condicionante tras el recojo de la información es trascendente, siendo la confirmación de aquello la literatura y la contrastación para la compresión del concepto de la realidad emergente. De esta forma se llega a la hipótesis que las investigaciones cualitativas se fundamentan en el poder investigativo y la conceptualización de las manifestaciones de las personas en los fenómenos educativos de su momento.

La teoría emergente producto del estudio son: los fundamentos de las investigaciones cualitativas son las formas de conocer y hacer conocer la realidad educativa, mediante el poder investigativo y la conceptualización de las manifestaciones de las personas en los fenómenos educativos de su momento; para contribuir en el conocimiento profundo del problema investigativo cualitativo y solucionarlo o mejorarlo. 


\section{Referencias Bibliográficas}

Bénard, S. (2016). La teoría fundamentada. Una metodología cualitativa. In Journal of Petrology (Vol. 1, Issue 1). https://doi.org/10.1017/СBO9781107415324.004

Cerrón, W. (2014). Conocimiento y matrices epistémicas. Horizonte de la Ciencia, 4(6), 87. https://doi.org/10.26490/ uncp.horizonteciencia.2014.6.96

Klimovsky, G., Ziziemsky, D., Azcoaga, J., Bleger, J., García, L., Masotta, O., Musso, R., \& Ziziemsky, D. (1971). Métodos de investigación en psicología y psicopatología (N. Visión (ed.)).

Munarriz, B. (2001). Técnicas y métodos en Investigación cualitativa. 95, 155. https://doi.org/10.2307/40184354

Vanossi, J. R. (2003). Ciencias duras y ciencias blandas : ¿Compartimentos estancos o apoyaturas metodológicas? (pp. $1-14)$.

Vasilachis, I. (2006). Estrategias de investigación cualitativa. Gedisa, 2002, 1-22. https://doi.org/10.15713/ins.mmj.3 